\title{
MEAN VALUES, MOMENTS, MOMENT RATIOS AND A GENERALIZED MEAN VALUE THEOREM FOR SIZE DISTRIBUTIONS
}

\author{
"WILLI PABST, SOŇA HříBALOVÁ \\ Department of Glass and Ceramics, University of Chemistry and Technology, Prague, \\ Technická 5, 16628 Prague 6, Czech Republic \\ "E-mail: pabstw@vscht.cz
}

Submitted June 24, 2019; accepted August 27, 2019

\begin{abstract}
Keywords: Size distributions (number-, length-, surface-, volume-weighted), Mean size (superarithmetic, arithmetic, geometric, harmonic and subharmonic), Moments, Moment ratios, Herdan's theorem

Generalized mean values of size distributions are defined via the general power mean, using Kronecker's delta to allow for the geometric mean. Special cases of these generalized mean values are the superarithmetic, arithmetic, geometric, harmonic and subharmonic means of number-, length-, surface-, volume- and intensity-weighted distributions. In addition to these special cases, however, our generalized $r$-weighted k-mean allows for non-integer values of $k$, which can be an advantage for describing material responses or effective properties of heterogeneous materials or disperse systems that are determined in a different way by different parts of a size distribution. For these generalized mean values a theorem is proved, which contains Herdan's theorem as a special case and turns out to be identical to Alderliesten's symmetry relation for moment ratios. In contrast to the moment-ratio notation, however, the interpretation of our notation is simple, intuitive and self-evident.
\end{abstract}

\section{INTRODUCTION}

Size distributions of particles, droplets, bubbles, inclusions and pores play an important role in many fields of science, including materials science. For example, the size distributions of dust particles or water droplets in the atmosphere of the Earth, particles or droplets of immiscible liquids in liquid media, i.e. suspensions and emulsions, second-phase particles or pores in transparent ceramics, all determine the effects of light scattering in these disperse media and heterogeneous materials. Size distributions contain the maximum amount of size information available. In practice, however, it is desirable to reduce this amount to the minimum amount of information necessary, ideally to one number (a mean size), that represents the whole size distribution for the purpose of phenomena explanation, process control or property calculation. Of course, there is an infinite number of mean sizes, and the selection of the appropriate mean for a certain phenomenon, process or property is a question of the underlying theory. For all these purposes, however, a rational terminology and notation must be available for mean sizes.

Probably the most important milestones in this field are Herdan's classical book on Small Particle Statistics
[1], the German school of particle sizing [2-4] and Alderliesten's profound and authoritative treatment of mean particle diameters [5-12]. The latter has clearly emphasized the insufficiency of the German DIN 66141 [13] and European ISO 9276-2 [14] notation, which is based on the moment notation, and has proposed an alternative in the form of the so-called moment-ratio notation, which has been the basis of the British standard BS 2955 [15]. We fully agree with Alderliesten's criticism of mean values based on the moment notation. In particular, the fact that geometric means cannot be defined within this framework, is indeed a servere deficiency of moment-based mean values. More than that, the German school of particle sizing has introduced a great deal of unnecessary confusion into particle sizing terminology by not clearly distinguishing between volume-weighted distributions (of particle size measures, e.g. equivalent diameters) from volume distributions (i.e. numberweighted distributions of particle volumes). Of course, in terms of any rational definition of size, which always has dimensions of length, the latter should not be called a size distribution at all. The same argumentation holds for surface-weighted distributions, for example. Repeatedly we have come across the opinion that this criticism concerns only some early papers and that most 
current authors use the correct term "volume-weighted distributions" instead of "volume distributions", but this standpoint ignores the fact that volume distributions do exist (e.g. as the primary result of Coulter counter measurements) and are closely related to volumeweighted distributions. In spite of all these justified points of criticism, however, it has to be admitted that Alderliesten's moment-ratio notation is somewhat awkward, because the meaning of the indices is not intuitively clear and can be understood only after considerable training.

In this context it has to be recalled that in other fields of engineering science, e.g. the theory of multiphase materials, generalized mean values are defined without any recourse to moments [16-18]. In the traditional formalism this definition consists of two parts: the definition of a general power mean and an extra definition for the geometric mean (which is included in the general power mean only asymptotically and has no equivalent at all in the moment notation). This is the usual way e.g. to extract specific mixture rules (e.g. the volume-weighted arithmetic or harmonic mean) from the generalized mean values (which may be considered as a general mixture rule). In any case the quantity to be averaged (e.g. an elastic modulus, thermal conductivity etc.) and also the type of weighting (e.g. mass- or volume-weighting) is clearly given and no one would have the idea to consider generalized mean values for squares or cubes of these quantities (e.g. the square of an elastic modulus or the cube of a thermal conductivity). In the same sense, it should be clear that size distributions, whatever their statistical weighting on the ordinate (e.g. number-weighted, length-weighted, surfaceweighted or volume-weigthted) are distributions of length measures (not surface or volume measures) on the abscissa.

In the present paper we show that the aforementioned two parts of the definition of generalized mean values can be formally combined into one definition by using Kronecker's delta to include the singular case of geometric means. Further we propose to use this definition as the fundamental definition of mean values of discrete particle size distributions, without any recourse to moments (it has to be noted that all computer-based representations of size distributions and all ensuing calculations of mean values are based on discrete distributions and that continuous distributions, which are the basis of the moment notation and Mellin transforms, are just idealizations that allow a formal foundation of the mean value definition by coupling it to the more basic concept of moments; for real size distributions, which are always discrete, such a formalization is unnecessary). In this way the intuitive notation of the German school can be retained, but without their traditional ballast of terminological inconsistencies and relics from the moment notation. This leads to a crystal-clear, simple and intuitive index notation for mean values. Moreover, in this paper we show that the fact that some of these mean values can be identical is not due to a redundancy in the German notation, as surmised by Alderliesten [5], but the consequence of a non-trivial theorem, equivalent to Alderliesten's symmetry relation for moment ratios, whose general form is presented here for the first time in DIN/ISO notation.

\section{THEORETICAL}

\section{Classification of size distributions}

Objects like particles, droplets, bubbles, inclusions and pores can be characterized by their size (in the present paper denoted as $x$ ). Size is a measure with dimensions of length [m], usually an equivalent diameter [19], for example the diameter of a (hypothetical) sphere that has some geometric characteristic or physical property in common with the object in question (which is usually of nonspherical shape). As trivial as it seems, it should be emphasized that if we are talking about size distributions, we are talking about distributions of these length measures, not surfaces or volumes. Of course, as mentioned in the introduction, also surfaces and volumes can have their distributions, but distributions of these quantities should not be called size distributions. Unfortunately, this simple rule of correct scientific language is not always obeyed, which constantly leads to a great deal of confusion in the literature. In particular, the current ISO standard [14], which is based on the old German industry standard [13], has contributed a major part to this confusion and has therefore been justly criticized by Alderliesten [5-10]. Not only beginners and newcomers in the field, but also many authors as well as companies producing particle size equipment and utilizing particle sizing software seem to be confused by this misleading terminological tradition.

Disperse systems (including heterogeneous materials with matrix-inclusion microstructure) containing particles, droplets, bubbles, inclusions or pores of only one size are called monodisperse. However, the majority of real disperse systems is polydisperse, i.e. exhibits a size distribution of finite width. Such a size distribution can be represented in discrete form as a histogram or in continuous form as a curve, both either as a probability density graph (here denoted as $q$ ) or as a cumulative graph (here denoted as $Q$ ). The size distribution is called monomodal when its probability density graph (histogram or curve) exhibits one maximum (so-called mode) or polymodal (also called multimodal) when its probability density graph exhibits more than one mode, with two modes called bimodal, with three modes trimodal etc. The mode, i.e. the maximum in the probability density graph, corresponds to a point of inflection in the cumulative graph. Apart from the modes, also quantiles are useful characteristic values. The most important of these is the so-called median, i.e. the size value, below and above which there is exactly $50 \%$ of 
the overall amount (either their overall number or the sum of all diameters, all surfaces or all volumes etc.) of all objects. The most fundamental size distribution is number-weighted (denoted $q_{0}$ or $Q_{0}$ ). It is the primary results e.g. of microscopic image analysis and the input information e.g. for calculating optical transmittance. On the other hand, the majority of common methods for size distribution measurements does not provide a number-weighted distribution directly, but most often volume-weighted distributions $\left(q_{3}\right.$ or $\left.Q_{3}\right)$, and in these cases it is necessary to obtain the number-weighted distribution via transformation from the (usually volume-weighted) primary data [20, 21]. For example, laser diffraction yields volume-weighted distributions as primary results [22, 23]. Much less common are length-weighted $\left(q_{1}\right.$ or $\left.Q_{1}\right)$ or surface-weighted $\left(q_{2}\right.$ or $Q_{2}$ ) size distributions. A rather abstract, but in practice quite important, type of size distribution is a so-called intensity-weighted size distribution $\left(q_{6}\right.$ or $\left.Q_{6}\right)$, which is the primary result of dynamical light scattering or photon correlation spectroscopy [23].

General power means, expectation values, moments and moment ratios

When the size distribution is given in discrete form (i.e. as a histogram), the general power mean of a size measure $x$, e.g. an equivalent sphere diameter, can be defined as

$$
\bar{x}_{k, r}=\left(\frac{\sum x_{i}^{k+\mathrm{r}} n_{i}}{\sum x_{i}^{r} n_{i}}\right)^{1 / k},
$$

where $k$ is an integer number denoting the type of average (e.g. arithmetic mean for $k=+1$ and harmonic mean for $k=-1$ ) and $\mathrm{r}$ is another integer number denoting the type of distribution (e.g. number-weighted for $r=0$, length-weighted for $r=1$, surface-weighted for $r=2$ and volume-weighted for $r=3$ ) etc. Since in this definition $k$ cannot be zero, the geometric mean is usually defined by the additional relation

$$
\bar{x}_{k, r}=\exp \frac{\sum x_{i}^{r} n_{i} \ln x_{i}}{\sum x_{i}^{r} n_{i}} .
$$

In these equations $x_{i}$ denotes the size fraction (more precisely the midpoint of the size fraction or histogram bin), $n_{i}$ the number of objects in this size fraction (histogram bin), and here and in the sequel summation is from the minimum size to the maximum size. Strictly speaking, these are definitions of estimators, because the true size distribution is not necessarily represented by the midpoints of size fractions. It should be noted that without Equation 2 the definition of general mean values is not complete.

Herdan's theorem [1] states that the harmonic mean of an $r$-weighted distribution equals the arithmetic mean of a $(r-1)$-weighted distribution, i.e.

$$
\bar{x}_{-1, r}=\left(\frac{\sum x_{i}^{r-1} n_{i}}{\sum x_{i}^{r} n_{i}}\right)^{-1}=\frac{\sum x_{i}^{r} n_{i}}{\sum x_{i}^{r-1} n_{i}}=\bar{x}_{1, r-1} .
$$

A special case of this theorem is the well-known fact that the harmonic mean of a volume-weighted distribution is equal to the harmonic mean of the surfaceweighted distribution. While all materials scientists know that small particle size implies higher particle surface, it is not known to all that it is the harmonic mean of the volume-weighted size distribution, and nothing else, that is inversely proportional to the specific surface. This equality (Herdan's theorem) is a non-trivial result that has been unjustly criticized by Alderliesten [5], who interpreted it incorrectly as mere redundancy of the German DIN notation.

The expectation value of a random variable $x$, e.g. a size measure, is generally defined as

$$
E(x)=\int_{0}^{\infty} x q_{0}(x) d x,
$$

where $q_{0}(x)$ is a number-weighted probability density distribution. When the random variable is replaced by a function (of the random variable) the expectation value is

$$
E[g(x)]=\int_{0}^{\infty} g(x) q_{0}(x) d x,
$$

and when the function is chosen to be $g(x)=x^{k}$, the expectation value

$$
E\left(x^{k}\right)=\int_{0}^{\infty} x^{k} q_{0}(x) d x=M_{k},
$$

is called the $k$-th general moment, which can also be considered as a Mellin transform of the number-weighted probability density curve (frequency curve). The zeroth general moment $M_{0}$ is equal to unity (normalization condition), and the first general moment $M_{1}$ is the arithmetic mean. Central moments are obtained when the function $x^{k}$ is replaced by the function $\left(x-M_{1}\right)^{\mathrm{k}}$.

For other than number-weighted distributions weighted moments are used. In particular, the weighted general moments are

$$
M_{k, r}=\int_{0}^{\infty} x^{k} q_{r}(x) d x=\int_{0}^{\infty} x^{k+r} q_{0}(x) d x .
$$

In terms of size fractions $x_{i}$ and their frequencies $n_{i}$ the weighted general moments of discrete size distributions can be written as

$$
M_{k, r}=\frac{\sum x_{i}^{k+r} n_{i}}{\sum x_{i}^{r} n_{i}} .
$$

A fundamental relation between different moments, which can be used for interpreting the physical meaning of a given moment, is

$$
M_{k, r}=\frac{M_{k+r, 0}}{M_{r, 0}}=\frac{M_{k+r-e, e}}{M_{r-e, e}} .
$$


Pabst W., Hř́balová S.

Using this relation, Herdan's theorem can be written in moment notation as

$$
M_{-1, r}=\left(M_{1, r-1}\right)^{-1} .
$$

The general power means introduced via Equation 1 are related to the moments via the equation

$$
\bar{x}_{k, r}=\sqrt[k]{M_{k, r}},
$$

where $M_{k, r}$ is the $k$-th general moment of the $r$-weighted distribution. Note that only the first general moments have units of length [m], while zeroth general moments are dimensionless [1], second general moments have units of length squared $\left[\mathrm{m}^{2}\right]$ etc. Moreover, obviously, for $k=0$ this expression becomes undefined, because roots of zeroth order are not defined, so that geometric means actually have no equivalent in the moment notation. Both facts are serious and principal disadvantages of the moment notation and have been justly criticized by Alderliesten [5]. Unfortunately, in the current ISO standard [14] and the German industry standard [13], the general power means given by Equation 1 are coupled to moments via Equation 11, without taking into account that a complete definition of general mean values requires also Equation 2. Thus we completely agree with Alderliesten's criticism of moments (and the related ISO and DIN standards), but we would not agree with extending this criticism to the general mean values as such.

As mentioned above, compared to general power means and geometric means of size distributions, which all have constant units of length $([\mathrm{m}])$, moments of size distributions have the disadvantage of having varying units, depending on the order of the moment (e.g. [1], $[\mathrm{m}],\left[\mathrm{m}^{2}\right]$ etc.). This deficiency is avoided by moment ratios. In the so-called moment-ratio notation, used in the British standard 2955 [15] and elaborated by Alderliesten [5-12], the mean values $((p-s)$-means of $s$-weighted distributions) are written as

$$
D_{p, s}=\left(\frac{M_{p, 0}}{M_{s, 0}}\right)^{\frac{1}{p-s}}=\left(\frac{\sum x_{i}^{p} n_{i}}{\sum x_{i}^{s} n_{i}}\right)^{\frac{1}{p-s}},
$$

when $p \neq s$ with $p \geq s \geq 0$ and

$$
D_{p, s}=\exp \frac{\sum x_{i}^{p} n_{i} \ln x_{i}}{\sum x_{i}^{p} n_{i}}=\exp \frac{\sum x_{i}^{s} n_{i} \ln x_{i}}{\sum x_{i}^{s} n_{i}}
$$

when $p=s$ (geometric means of $s$-weighted distributions). It can be shown that moment ratios are related to our general mean values via the equation

$$
\bar{x}_{k, r}=D_{k+r, r} .
$$

In contrast to Equation 11 this equation does not exclude geometric means.

\author{
Generalized mean values of \\ size distributions and generalized \\ meanvalue theorem
}

Definition: We define a generalized $r$-weighted $k$-mean as

$$
\begin{aligned}
\bar{x}_{k, r} & =\left(1-\delta_{k 0}\right)\left(\frac{\sum x_{i}^{r} n_{i} x_{i}^{k}}{\sum x_{i}^{r} n_{i}}\right)^{1 / k}+\delta_{k 0} \cdot \exp \frac{\sum x_{i}^{r} n_{i} \ln x_{i}}{\sum x_{i}^{r} n_{i}}, \\
& =\left(1-\delta_{k 0}\right)\left(\frac{\sum x_{i}^{r+k} n_{i}}{\sum x_{i}^{r} n_{i}}\right)^{1 / k}+\delta_{k 0} \cdot \exp \frac{\sum x_{i}^{r} n_{i} \ln x_{i}}{\sum x_{i}^{r} n_{i}}
\end{aligned}
$$

where $x$ is the size measure chosen (typically an equivalent sphere diameter), $x_{i}$ the size fraction $i$ (usually the mid-point of the corresponding histogram bin) $i, n_{i}$ the number of objects in this bin, the summation symbol denotes the summation over all non-empty bins (or principally from 0 to $\infty$ ) and $\delta_{k 0}$ is the normalized delta distribution (Kronecker's delta) defined as

$$
\delta_{k 0}=\left\{\begin{array}{lll}
1 & \text { if } & k=0 \\
0 & \text { if } & k \neq 0
\end{array} .\right.
$$

Depending on the type of distribution, the value of $r$ is either 0 (number-weighted distribution), 1 (lengthweighted distribution), 2 (surface-weighted distribution), 3 (volume-weighted distribution) or 6 (so-called intensity-weighted distribution, which occurs e.g. in dynamic light scattering when the particles are small enough for the Rayleigh approximation to apply, in which the intensity of scattered light is proportional to the square of the polarizability, i.e. the particle volume). We are not aware of any other types of distributions and $r$ values that would be of practical importance, but of course our notation can be extended to other cases as well.

The value of $k$ determines the type of mean value. Special cases are the arithmetic mean $(k=1)$, the geometric mean $(k=0)$ and the harmonic mean $(k=-1)$. Beyond these types of means we have the quadratic mean $(k=2)$, the cubic mean $(k=3)$ etc., means with $k>1$ being generally denoted as superarithmetic means. Similarly, we have the first subharmonic mean $(k=-2)$, the second subharmonic mean $(k=-3)$ etc. For all these $k$-means the following majority relation holds:

$\ldots \bar{x}_{-3, r} \leq \bar{x}_{-2, r} \leq \bar{x}_{-1, r} \leq \bar{x}_{0, r} \leq \bar{x}_{1, r} \leq \bar{x}_{2, r} \leq \bar{x}_{3, r} \ldots$.

Note, however, that - in contrast to more traditional definitions - in our definition of generalized means the value of $k$ need not necessarily be an integer value. The rationale behind this is that in cases where small, medium-sized and large objects in a size distribution determine an effective property value to a different degree, e.g. optical transmittance of disperse systems (which is for small particles determined by Rayleigh scattering, for medium-sized particles by Mie scattering and for large particles by Fraunhofer diffraction), noninteger values of $k$ may be more appropriate for repre- 
senting the overall response of the material or disperse system.

It should be emphasized that for each type of distribution (i.e. each value of $r$ ) there is a different set of these mean values. Moreover, in addition to the aforementioned classical majority relation, also another majority relation holds for differently weighted distributions:

$$
\ldots \bar{x}_{k, 0} \leq \bar{x}_{k, 1} \leq \bar{x}_{k, 2} \leq \bar{x}_{k, 3} \leq \bar{x}_{k, 6} \ldots .
$$

Based on the aforementioned definition we can formulate the following:

Theorem: The $(-k)$-mean of an $(r+k)$-weighted distribution equals the $k$-mean of the corresponding $r$-weighted distribution, i.e.

$$
\bar{x}_{-k, r+k}=\bar{x}_{k, r} .
$$

Proof: Using the definition, Equation 15, and inserting $-k$ and $r+k$ for $k$ and $r$ we obtain

$\bar{x}_{-k, r+k}=$

$=\left(1-\delta_{-k 0}\right)\left(\frac{\sum x_{i}^{r+k-k} n_{i}}{\sum x_{i}^{r+k} n_{i}}\right)^{-1 / k}+\delta_{-k 0} \cdot \exp \frac{\sum x_{i}^{r+k} n_{i} \ln x_{i}}{\sum x_{i}^{r+k} n_{i}}$

$=\left(1-\delta_{-k 0}\right)\left(\frac{\sum x_{i}^{r} n_{i}}{\sum x_{i}^{r+k} n_{i}}\right)^{-1 / k}+\delta_{-k 0} \cdot \exp \frac{\sum x_{i}^{r+k} n_{i} \ln x_{i}}{\sum x_{i}^{r+k} n_{i}}$,

$=\left(1-\delta_{-k 0}\right)\left(\frac{\sum x_{i}^{r+k} n_{i}}{\sum x_{i}^{r} n_{i}}\right)^{1 / k}+\delta_{-k 0} \cdot \exp \frac{\sum x_{i}^{r+k} n_{i} \ln x_{i}}{\sum x_{i}^{r+k} n_{i}}=\bar{x}_{k, r}$ q.e.d.

Corollary 1: The special case $k=-k$ can occur only for $k=0$ (geometric mean). Therefore, for the geometric mean our theorem reduces to a trivial identity (in other words, $k=-k$, holds if and only if the $k$-mean is the geometric mean). Our theorem provides nontrivial results only if $k \neq 0$. It concerns mean values symmetrically distributed around the geometric mean, i.e. $k$-mean pairs for which the $\mathrm{k}$-values are +1 and -1 , +2 and $-2,+3$ and -3 etc.

Corollary 2: A special case of our theorem is the statement that the harmonic mean of an $r$-weighted distribution equals the arithmetic mean of an $(r-1)$-weighted distribution (Herdan's theorem [1]), or, more specifically, that the harmonic mean of a volume-weighted distribution $(r=3)$ equals the arithmetic mean of a surface-weighted distribution $(r=2)$. This special case is well known.

\section{Connection to other formalisms}

The formalism used in this paper is classical and consistent in itself. It can be readily connected to the moment notation (German DIN standard and European ISO standard) as well as the moment-ratio notation (British standard). The connection to the moment notation is given by Equation 11. Using the moment notation our mean value theorem can be written as

$$
\mathrm{M}_{k, r}=\left(\mathrm{M}_{-k, r+k}\right)^{-1} \text {. }
$$

On the other hand, the connection to the moment-ratio notation is given by Equation 14, and in this notation our mean value theorem adopts the nicely symmetric form

$$
D_{p, s}=D_{s, p},
$$

where we have redefined the indices as $p=k+r$ and $s=r$. Actually this symmetry relation for moment ratios has been discovered some time ago by Alderliesten [5]. However, Alderliesten interpreted the existence of this symmetry relation as an argument against the German DIN notation of mean diameters. For example, the fact that both $\bar{x}_{-1,3}$ and $\bar{x}_{1,2}$ are identical to $\bar{D}_{3,2}$ has been invoked as an example to demonstrate the redundancy of the German DIN notation, which "may hamper the physical interpretation of mean particle diameters" [5]. However, as mentioned above, the identity $\bar{x}_{-1,3}=\bar{x}_{1,2}$ is a special case of Herdan's theorem and an even more special case of our generalized mean value theorem. Far from being redundant and "hampering physical interpretation" it is the only way to understand, why the harmonic mean of the volumeweighted size distribution (and no other type of mean value) is inversely proportional to the surface density (i.e. the specific surface multiplied by the density) without any shape factor: this is the case only because the harmonic mean of the volume-weighted distribution happens to be (due to Herdan's theorem) the arithmetic mean of the surface-weighted distribution. The theorem presented here is just a generalization of Herdan's theorem.

In contrast to the moment notation, the momentratio notation allows the definition of geometric means and thus the formulation of generalized moment ratios in the form

$D_{p, s}=\left(1-\delta_{p s}\right)\left(\frac{\sum x_{i}^{p} n_{i}}{\sum x_{i}^{s} n_{i}}\right)^{1 /(p-s)}+\delta_{p s} \cdot \exp \frac{\sum x_{i}^{p} n_{i} \ln x_{i}}{\sum x_{i}^{p} n_{i}}$,

i.e. in the same way as our generalized $r$-weighted $k$-means, and, of course, in principle also the momentratio notation could be generalized to non-integer values of $p$. Thus the two notations are completely equivalent or, in other words, there is a one-to-one correspondence between the two, our notation being just more intuitive. In the moment-ratio notation negative values of $s=r$ would be required to calculate the harmonic mean of number-weighted distributions (or subharmonic means of distributions weighted with $r \leq 1$ ). Actually, in the moment-ratio notation the harmonic mean of a numberweighted distribution is formally calculated as $D_{0,-1}$, i.e.an arithmetic mean of a distribution with weighting $r=-1$, which would be a distribution weighted by inverse lengths, a rather awkward interpretation indeed. 
Therefore the use of negative indices is usually avoided in the moment-ratio notation, although there is no justification for this omission on physical grounds. Our notation avoids all these problems, and the harmonic means of number-weighted distributions, as well as the subharmonic means of distributions weighted with $r \leq 1$, follow in a completely natural way. Moreover, in contrast to the moment-ratio notation, our notation allows a much simpler and much more intuitive interpretation of the respective mean values, the meaning of which is directly evident from the indices.

\section{CONCLUSION}

Generalized mean values of size distributions, here called generalized $r$-weighted $k$-means, have been defined via the general power mean, using Kronecker's delta to allow for the geometric mean. In this way the two original defining equations are combined into a single equation, without any direct recourse to moments. Special cases of these generalized mean values are the superarithmetic, arithmetic, geometric, harmonic and subharmonic means of number-, length-, surface-, volume- and so-called intensity-weighted distributions. In addition to these special cases, however, our generalized $r$-weighted $k$-mean allows for non-integer values of $k$, which can be an advantage for describing material responses or effective properties of heterogeneous materials or disperse systems that are determined in a different way by different parts of a size distribution (e.g. optical transmittance, which is principally determined by the whole size distribution, but is affected differently by small, medium-sized and large dispersed particles, inclusions or pores). For these generalized mean values a theorem has been proved, which contains Herdan's theorem as a special case and turns out to be identical to Alderliesten's symmetry theorem for moment ratios. In contrast to the moment notation and the moment-ratio notation, however, the interpretation of our notation is simple, self-evident and intuitive.

\section{Ackowledgement}

This work is part of the project "Partially and fully sintered ceramics - processing, microstructure, properties, modeling and sintering theory" (GA18-17899S), supported by the Czech Science Foundation (GAČR).

\section{REFERENCES}

1. Herdan G. (1960). Small Particle Statistics. Second revised edition. Butterworths.

2. Rumpf H., Ebert K. F. (1964): Darstellung von Korn- grössenverteilungen und Berechnung der spezifischen Oberfläche. Chemie Ingenieur Technik, 36, 523-537. doi: 10.1002/cite. 330360516

3. Leschonski K., Alex W., Koglin B. (1974): Darstellung und Auswertung von Teilchengrössenanalysen. Chemie Ingenieur Technik, 46, 23-26, 101-106. doi: 10.1002/cite. 330460105

4. Leschonski K. (1984): Representation and evaluation of particle size analysis data. Particle \& Particle Systems Characterization, 1, 89-95. doi: 10.1002/ppsc.19840010115

5. Alderliesten M. (1990): Mean particle diameters. Part I. Evaluation of definition systems. Particle \& Particle Systems Characterization, 7, 233-241. doi: 10.1002/ppsc. 19900070138

6. Alderliesten M. (1991): Mean particle diameters. Part II. Standardization of nomenclature. Particle \& Particle Systems Characterization, 8, 237-241. doi: 10.1002/ppsc. 19910080143

7. Alderliesten M. (2002): Mean particle diameters. Part III. An empirical evaluation of integration and summation methods for estimating mean particle diameters from histogram data. Particle \& Particle Systems Characterization, 19, 373-386. doi: 10.1002/ppsc. 200290000

8. Alderliesten M. (2004): Mean particle diameters. Part IV. Empirical selection of the proper type of mean particle diameter describing a product or material property. Particle \& Particle Systems Characterization, 21, 179-196. doi: $10.1002 / p p s c .200400917$

9. Alderliesten M. (2005): Mean particle diameters. Part V. Theoretical derivation of the proper type of mean particle diameter describing a product or material property. Particle \& Particle Systems Characterization, 22, 233-245. doi: $10.1002 /$ ppsc. 200500987

10. Alderliesten M. (2010): Mean particle diameters. Part VI. Fundamental distinction between statistics based (ISO/DIN) and physics based (moment-ratio) definition systems. Particle \& Particle Systems Characterization, 27, 7-20. doi: 10.1002/ppsc. 201000002

11. Alderliesten M. (2013): Mean particle diameters. Part VII. The Rosin-Rammler size distribution: physical and mathematical properties and relationships to momentratio defined mean particle diameters. Particle \& Particle Systems Characterization, 30, 244-257. doi: 10.1002/ppsc. 201200021

12. Alderliesten M. (2005): Mean particle diameters - from statistical definition to physical understanding. Journal of Biopharmaceutical Statistics, 15, 295-325. doi: 10.1081/ BIP-200048774

13. DIN 66141: Darstellung von Korn-(Teilchen-)grössenverteilungen. 1974

14. ISO 9276-2: Representation of Results of Particle Size Analysis. Part 2. Calculation of Average Particle Sizes/ Diameters and Moments from Particle Size Distributions. 2001.

15. BS 2955: Glossary of Terms Relating to Particle Technology. 1993.

16. Pabst W., Gregorová E. (2004): Effective elastic properties of alumina-zirconia composite ceramics - Part II: Micromechanical modeling. Ceramics-Silikáty, 48, 14-23.

17. Ji S. C., Wang Q., Xia B., Marcotte D. (2004): Mechanical properties of multiphase materials and rocks - a phenomenological approach using generalized means. Journal 
of Structural Geology, 26, 1377-1390. doi: 10.1016/j.jsg. 2003.12.004

18. Ji S. C., Gu Q., Xia B. (2006): Porosity dependence of mechanical properties of solid materials, Journal of Materials Science, 41, 1757-1768. doi: 10.1007/s10853-006-2871-9

19. Allen T. (1997). Particle Size Measurement (2 vols.). Chapman \& Hall.

20. Pabst W., Berthold C., Gregorová E. (2006): Size and shape characterization of polydisperse short-fiber systems. Journal of the European Ceramic Society, 26, 1121-1130. doi: 10.1016/j.jeurceramsoc.2005.01.053
21. Pabst W., Berthold C., Gregorová E. (2007): Size and shape characterization of oblate and prolate particles, Journal of the European Ceramic Society, 27, 1759-1762. doi: 10.1016/j.jeurceramsoc.2006.05.076

22. Pabst W., Kuneš K., Havrda J., Gregorová E. (2000): A note on particle size analyses of kaolins and clays. Journal of the European Ceramic Society, 20, 1429-1437. doi: 10.1016/S0955-2219(00)00016-9

23. Xu R. (2000). Particle Characterization: Light Scattering Methods. Kluwer Academic Publishers. 